\title{
L’université Camerounaise Face Aux Mouvements De Revendications Identitaires
}

\section{[Cameroon's universities and identity-based movements]}

\author{
Mama Chandini* \\ Départment des Sciences de l'Education, Ecole Normale Supérieure de Bertoua, Université de Ngaoundéré, \\ Cameroun
}

*Corresponding Author: Mama Chandini, Départment des Sciences de l'Education, Ecole Normale Supérieure de Bertoua, Université de Ngaoundéré, Cameroun

\begin{abstract}
In recent years, Cameroon has witnessed a rise in identity-based withdrawals and a resurgence of communitarian demands. Cameroonian universities do not seem to escape this reality of multicultural societies, which poses the problem of cohabitation and integration of the different socio-cultural components in this country. In this context, the present contribution aims at answering the question of whether the integrationist discourse regularly conveyed by the public authorities allows Cameroonian universities to escape the trap of segregationist tendencies. The objective of this paper is to determine the effects of the interventions of communitarian movements on the teaching/learning conditions in Cameroonian universities. In order to do so, a detailed analysis of institutional, individual or collective discourses, attitudes and practices allows us to highlight two contradictory trends: an official discourse advocating the virtues of national integration on the one hand, and individual or collective attitudes revealing identity-based cleavages on the other. This leads to the conclusion, in the light of concrete illustrations, that identity-based withdrawal and communitarian demands constitute threats to the dynamics of socio-cultural integration of students and lecturers in Cameroonian universities, which affects teaching and learning conditions.
\end{abstract}

Keywords: Identity-based movements; Teaching and learning conditions; Universities; Cameroon.

\section{INTRODUCTION}

Depuis plus d'une décennie, on assiste au Cameroun à une montée de revendications à caractère identitaire. Ces revendications, portées par les élites régionales, ont une apparence sociopolitique et recèlent au fond des soubassements communautaristes. C'est ainsi qu'il ne se passe plus de temps sans que l'on enregistre des récriminations, des dénonciations, et des revendications en provenance de groupes sociologiques qui s'estiment «marginalisées » dans le processus de développement social. Dans la même lancée, des regroupements à vocation communautariste ne cessent de se former avec pour crédo la réclamation d'une considération plus accrue de leurs spécificités socioculturelles ou de leur poids politique au sein de la société camerounaise. Ces revendications sont dirigées vers les pouvoirs publics et plus particulièrement à l'attention de la plus haute autorité politico-administrative du Cameroun, notamment le Président de la République. Les requêtes qui y sont formulées se résument officiellement en une doléance : les interventions en vue d'une meilleure prise en compte des filles et fils des communautés concernées dans le partage du «gâteau national ». En clair, il s'agit des réclamations visant l'obtention d'une part accrue des privilèges au sein de l'appareil de l'Etat ou dans divers secteurs de la vie publique à travers l'octroi de positions avantageuses au bénéfice des communautés revendicatrices.

Plus prosaïquement, les élites régionales se constituent généralement au sein d'associations communautaristes avec pour agenda la sollicitation, de la part du Président de la République, de l'octroi de faveurs ou de facilités aux membres de leurs communautés. Ces demandes de privilèges visant à ce que ces derniers accèdent soit aux hautes fonctions administratives, soit à l'intégration dans des écoles de formation pour lesquelles l'entrée se fait sur concours, soit encore à l'érection d'infrastructures sociales dans leur région d'origine.

Cette réalité des sociétés multiculturelles, qui touche la quasi-totalité des pans de la vie nationale, ne cesse de s'infiltrer subtilement dans les arcanes de l'université camerounaise. En effet, les élites 
régionales au Cameroun, constituées en véritables groupes de pression, ont régulièrement tendance à élaborer des programmes dont l'objet vise à exercer une influence sur l'orientation des politiques institutionnelles dans ce pays. Cette influence a des ramifications qui vont jusqu'à toucher le vécu des institutions universitaires. Or lorsqu'on sait que l'université, à travers les âges, est considérée comme un sanctuaire ${ }^{1}$, lorsqu'on sait combien l'enseignement supérieur fait l'objet de convoitises ${ }^{2}$ et quand on prend en compte l'extrême fragmentation identitaire de la société camerounaise, on peut s'inquiéter de l'immixtion fréquente des mouvements de revendication à caractère régionaliste dans la gestion des universités.

Compte tenu des considérations ainsi énoncées, on peut se poser la question de savoir si le discours intégrationniste régulièrement véhiculé par les pouvoirs publics permet aux universités camerounaises d'échapper au piège des velléités ségrégationnistes, caractérisées par les replis identitaires et les revendications régionalistes. Le problème qui se pose est essentiellement celui de savoir à quel degré les interventions des mouvements de revendication communautaristes, qui prennent de plus en plus une envergure sociopolitique, affectent les conditions d'enseignement/apprentissage en milieu universitaire au Cameroun.

L'objectif de la présente contribution est de déterminer les effets des interventions des mouvements de revendication à caractère communautariste sur les conditions d'enseignement/apprentissage dans les universités camerounaises.

\section{APPROCHE METHODOLOGIQUE}

Cette étude est une recherche qualitative de type exploratoire. Les données qui y sont exploitées ont été collectées dans le cadre du projet d'un ouvrage collectif commandité par l'Université de Maroua et paru en $2020^{3}$. A cet égard, la technique du choix raisonné a permis de soumettre un échantillon de 230 sujets à l'entretien semi-directif, car cette technique «permet d'identifier la représentativité des personnes et des situations en fonction de leur expérience de l'évènement que l'on veut étudier » [1].

Elle essaie d'analyser la question à travers le décryptage des attitudes, l'analyse des discours et une lecture des pratiques quotidiennes des acteurs. Dans cette logique, l'observation directe des pratiques sociétales, la recherche documentaire et l'interview réalisées auprès de 120 étudiants (dont 15 étudiants dans chacune des huit (08) universités d'Etat du Cameroun), de 80 responsables académiques (à raison de 10 par université d'Etat) et de 30 acteurs sociopolitiques (en provenance de toutes les 10 Régions du Cameroun) permettront d'identifier ce qui peut constituer les véritables raisons de l'irruption constante des revendications à visée identitaire dans les universités camerounaises et les conséquences que ces intrusions peuvent avoir sur les conditions d'enseignement et d'apprentissage. A leur demande, les interventions des personnes interviewées sont recouvertes du sceau de l'anonymat. Une analyse de contenu [2] permettra le traitement des données ainsi recueillies.

\section{Resultats Et Discussion}

\subsection{Résultats}

\subsubsection{Structuration Socioculturelle De La Société Camerounaise}

En plus de ses deux langues officielles constituées du français et de l'anglais héritées de la colonisation, le Cameroun compte environ 250 groupes ethniques et autant de variétés linguistiques. Ses populations adhèrent à une multitude de confessions religieuses, croyances spirituelles et pratiques cultuelles. Elles sont réparties sur un ensemble de quatre grandes aires culturelles, à savoir l'aire Grassfield (les Régions de l'Ouest et du Nord-Ouest), l'aire Fang-Beti (les Régions du Centre, du Sud et de l'Est), l'aire Sawa (les Régions du Littoral et du Sud-Ouest) et l'aire soudano-sahélienne

\footnotetext{
${ }^{1}$ De ce fait l'université est considérée comme étant le temple du savoir : n'y ont accès que des personnes jouissant d'une haute valeur intellectuelle et morale.

${ }^{2} \mathrm{Du}$ fait du prestige social et de la plus-value économique qui y sont rattachés.

3 Bachir Bouba (Dir.). Education, formation et apprentissage et apprentissage en contexte multiculturel camerounais. Douala : Editions Cheikh Anta Diop.
} 
(les Régions du Nord, de l'Extrême-Nord et de l'Adamaoua). A ces groupes ethniques, culturels et religieux, il faut ajouter les «minorités », essentiellement constituées de pygmées, de Mbororos, de personnes vivant avec un handicap, de déplacés internes, d'immigrés et de réfugiés.

Toutes ces diversités sociologiques constituent autant de contradictions qu'il faut gérer en vue d'assurer un certain équilibre dans toutes les sphères de l'Etat, gage de l'intégration nationale, de la cohésion sociale et de la stabilité sociopolitique du pays. Dans ce contexte, le plus souvent marqué par la propension hégémonique de certains groupes socioculturels, les autres groupes s'estiment « lésés » dans la répartition des ressources du pays et, en conséquence, entreprennent des actions de revendication qui se manifestent sous diverses formes. Dans le cadre de la présente étude, nous nous intéressons à celles qui touchent particulièrement les universités camerounaises.

\subsubsection{Des Politiques Claires De Promotion De L'intégration Nationale Au Cameroun}

Conscient de sa diversité sociologique et de la nécessité de garantir la coexistence pacifique et la cohésion sociale au sein et entre les différentes communautés (ethniques, linguistiques, religieuses, etc.), le Cameroun a pris un certain nombre d'engagements et d'initiatives en vue de la promotion de l'intégration socioculturelle des diverses composantes communautaires. Parmi les initiatives, on peut mentionner l'élaboration de la Stratégie Camerounaise d'Intégration Nationale en 2015 d'une part, et la mise sur pied de la Commission Nationale pour la Promotion du Bilinguisme et du Multiculturalisme par décret présidentiel n²017/013 du 23 janvier 2017 d'autre part.

L'instauration et l'observation d'une Semaine Nationale du Bilinguisme représentent également un signal fort dans la politique d'inclusion socioculturelle au Cameroun. En effet, cette Semaine précède généralement la traditionnelle fête de la jeunesse célébrée le 11 février de chaque année. Elle constitue une occasion pour la jeunesse de montrer sa capacité à promouvoir la compréhension mutuelle entre les communautés d'expression française et d'expression anglaise.

La Journée internationale de la Langue Maternelle est aussi célébrée au Cameroun chaque 21 février. Cette journée permet de promouvoir la diversité linguistique et culturelle, ainsi que le multilinguisme et le multiculturalisme comme vecteurs d'intégration nationale au Cameroun.

L'observation et la célébration de la Journée internationale de la Francophonie, le 20 mars de chaque année, et de la Journée du Commonwealth (Commonwealth Day), le second lundi du mois de mars, quant à elles donnent l'occasion supplémentaire de magnifier l'appartenance du Cameroun à deux communautés d'intérêt ayant en partage ses deux langues officielles, notamment le français et l'Anglais.

Du point de vue du développement des curricula, on peut dire que l'instauration de l'enseignement des langues et cultures camerounaises dans le système éducatif camerounais est un palier important dans la promotion de l'intégration socioculturelle des étudiants.

Outre ces démarches qui visent à promouvoir l'intégration dans toutes les sphères de la société, des textes fondateurs, aussi bien à caractères généraux que spécifiques, positionnaient déjà l'intégration nationale au Cameroun comme la pierre angulaire de toute action publique. C'est ainsi que le préambule de la Constitution de 1996 stipule que : «Le peuple camerounais, fier de sa diversité linguistique et culturelle, élément de sa personnalité nationale qu'elle contribue à enrichir, mais profondément conscient de la nécessité impérieuse de parfaire son unité, proclame solennellement qu'il constitue une et même Nation, engagée dans le même destin et affirme sa volonté inébranlable de construire la patrie camerounaise sur la base de l'idéal de fraternité, de justice et de progrès ... »

La Loi n98/004 du 04 avril 1998 d'Orientation de l'éducation indique en son article 3 que «l'Etat consacre le bilinguisme à tous les niveaux d'enseignement comme facteur d'unité et d'intégration nationales ». Elle poursuit en son article 7 que «l'Etat garantit à tous l'égalité de chances d'accès à l'éducation sans discrimination de sexe, d'opinions politiques, philosophiques, religieuses, d'origine sociale, culturelle, linguistique ou géographique ».

Quant à la Loi ${ }^{\circ} 005$ du 16 avril 2001 portant orientation de l'enseignement supérieur, elle dispose en son article 5 que «l'Etat consacre le bilinguisme au niveau de l'enseignement supérieur comme facteur d'unité et d'intégration nationales ». On peut également voir que l'article 11 alinéa 1 (a) de ce document insiste sur le fait que «l'Etat protège contre toute discrimination tout postulant à 
l'enseignement supérieur, en raison de la race, du genre, de l'âge, de la religion, de l'origine linguistique et géographique ».

Compte tenu de la grande diversité ethnique et linguistique du Cameroun, la politique dite de l'équilibre régional constitue également un moyen visant l'intégration des différentes composantes socioculturelles dans différents espaces de ce pays. Le décret $n^{\circ} 82 / 407$ du 7 septembre 1982, modifiant et complétant celui du n $\mathrm{n}^{\circ} 75 / 496$ du 3 juillet 1975 prévoit en fait que la répartition des places, pour les concours d'entrée dans les grandes écoles débouchant sur l'intégration dans la Fonction publique par exemple, tienne compte de la «région d'origine» des parents [3]. En encourageant la représentativité de toutes les composantes sociologiques dans tous les domaines de la vie publique du pays, la politique de l'équilibre régional au Cameroun vise en définitive à promouvoir l'équité sociale et à prévenir les conflits interethniques [4].

En considération de l'engagement politique à promouvoir et à assurer l'intégration socioculturelle attesté dans les différentes positions officielles que nous venons de passer en revue, on peut se demander si ces propos intégrationnistes sur le brassage interculturel résistent aux velléités ségrégationnistes entreprises par certains acteurs sociaux ou par certains regroupements à caractère communautariste.

\subsubsection{Manifestations Des Revendications a Caractère Communautariste Autour Du Système Camerounais D'enseignement Supérieur}

Il convient de signaler que les mouvements de revendication identitaires se manifestent sous plusieurs formes au Cameroun. Ils commencent généralement par des regroupements communautaristes, s'en suivent des pétitions sous forme de mémorandum, puis des déclarations à travers les médias, les lettres ouvertes et les réseaux sociaux.

\subsubsection{Récurrence De Regroupements A Caractère Communautariste Dans La Société Camerounaise}

Dans l'agir quotidien des membres des différentes communautés ethniques au Cameroun, on note un regain d'inscription des actions dans une logique identitaire et régionaliste. C'est ainsi que les ressortissants des différentes aires culturelles s'organisent de plus en plus autour de regroupements à caractère communautariste. Il s'agit de ce qu'on appelle l'appartenance à « des groupes politisés » dont font partie les «associations d'élites régionales, des associations culturelles, comme le «Laakam » du groupe Bamiléké, «Essingan » du groupe Béti, «Mbock Lihaa » du groupe Bassa et Mpoo, «le Ngondo» du groupe Duala, «les élites du grand Nord», etc.» [5]. A cette liste, nous pouvons ajouter d'autres associations émergentes telles que «Ya-Kamata» du groupe Haoussa, « Nkoul-Bebend » du groupe Maka-Bebend ou «Mboscuda » du groupe Mbororo, et tout récemment, le «Mouvement 10000000 de Nordistes ». Les activités de ce dernier mouvement ont été frappées d'interdiction le 18 novembre 2020, par les pouvoirs publics camerounais à travers un communiqué radio-presse signé du ministre de l'Administration territoriale ${ }^{4}$.

Ces groupes d'appartenance qui ont investi l'espace public camerounais ces dernières années, ont une aura très étendue ${ }^{5}$. Ils constituent de véritables réseaux d'influence et de puissants groupes de pression au service de la défense exclusive des intérêts communautaristes. Ils représentent également une force de positionnement individuel pour certains de leurs leaders qui trouvent en ces regroupements un tremplin pour accéder à de hauts postes de responsabilité. On a vu que ces organisations s'illustrent généralement par la formulation de revendications multiformes au bénéfice des membres de leurs communautés respectives. Leur influence n'épargne pas le milieu universitaire, au regard des actions de lobbying qu'ils engagent à travers l'exercice de pressions sur les milieux de décision. Ces pressions se manifestent très souvent par des interventions pour que les ressortissants de leurs communautés d'appartenance puissent bénéficier de positions avantageuses en milieu universitaire, que ce soit en qualité d'étudiants, d'enseignants ou de responsables administratifs. «Au sein de ces associations,

\footnotetext{
${ }^{4}$ Le ministre de l'Administration territoriale accuse ce mouvement de mener les activités susceptibles de porter atteinte à l'intégration nationale du pays »

${ }^{5}$ Leur auréole s'étend généralement sur l'ensemble du territoire camerounais jusqu'au niveau de la diaspora.
} 
circulent souvent des listes pour l'inscription des noms des candidats, originaires de la région, ayant postulé à tel ou tel concours, en vue de l'intervention des élites pour qu'ils soient définitivement admis audit concours ", témoigne un étudiant. Cette attitude est révélatrice de l'injustice dont se plaignent certains candidats recalés aux concours d'entrée dans les grandes écoles au Cameroun. Ces candidats estiment que les ressortissants de certaines régions, en l'occurrence celles dont les élites sont proches du pouvoir politique, se trouvent «favorisés » au détriment de ceux issus des autres régions. Cet état de choses conduit à s'interroger sur la qualité des candidats définitivement admis en formation à l'université, sur leur capacité à pouvoir assimiler les enseignements qui leur seront dispensés et sur leur aptitude à venir à bout des apprentissages.

Il convient d'indiquer que ces mouvements sont organisés sous forme d'associations formelles, à l'instar de celles dont l'échantillon est présenté plus haut, mais peuvent également agir sous le couvert de l'anonymat avec des références à une communauté bien identifiable.

\subsubsection{La recrudescence des memoranda comme modus operandi des revendications à caractère communautariste}

Un nouveau canal de revendications à caractère identitaire a investi l'espace public en général et fait son entrée dans les milieux universitaires en particulier cette dernière décennie au Cameroun : il s'agit des memoranda. Il convient d'indiquer que la récurrence et l'intensité de cette activité épistolaire dans le pays sont l'émanation de l'activisme des élites qui, estimant que leur communauté mérite davantage de «réalisations », de «retombées politiques » ou leur "part de gâteau national », n'hésitent pas souvent à saisir les plus hautes autorités politico-administratives, généralement le Président de la République, pour se faire entendre. Ce phénomène est désigné sous le néologisme du «ma-partisme » [6], qui est un dérivé de l'expression nominale « ma part ». Il s'agit d'une situation de clientélisme qui décrit l'état de tout acteur social au Cameroun qui cherche prioritairement ses intérêts, qu'ils soient individuels ou d'ordre régionaliste, aux dépens de l'intérêt général. La question qu'il se pose généralement en pareille situation est la suivante : «Où est ma part? », pour dire «qu'est-ce que je gagne dans cette opération?».

C'est ainsi qu'on assiste ces derniers temps au Cameroun à la recrudescence de pétitions, initiées par les associations d'élites régionales, en direction du Chef de l'Etat, en vue de formuler et de poser les problèmes auxquels leurs régions ou les ressortissants de leurs régions sont confrontés. A ce sujet, ces dignitaires régionaux, constitués en véritables groupes de pression, font de plus en plus irruption dans les arcanes de l'Enseignement supérieur pour réclamer soit la création d'institutions universitaires dans leur région d'origine, soit la nomination de leurs «fils» à des postes de responsabilité à l'université, soit l'admission définitive de leurs «enfants » aux concours de recrutement au sein des grandes écoles implantées dans leur région ou ailleurs. Dans ce sillage, on se souvient encore de la polémique suscitée par le «Mémorandum des Elites du Grand Nord». A travers ce document, les dignitaires de cette région avaient saisi le Chef de l'Etat camerounais pour revendiquer que soit revu à la hausse le nombre de places accordées aux ressortissants de cette région «afin que leurs enfants soient admis à l'ENS ». Contre toute attente, on s'est aperçu que cette requête a eu gain de cause, car à la grande surprise de tous, le Ministre camerounais de l'Enseignement supérieur autorisait l'admission, à l'ENS de Maroua, de la totalité des candidats originaires des trois régions septentrionales du Cameroun, après la promulgation du résultat définitif du concours d'entrée à l'Ecole Normale Supérieure de 1'Université de Maroua en $2008^{6}$. Cette revendication aux relents purement identitaires, laissait subodorer la prééminence de facto des «enfants » ressortissants de la région du grand Nord sur ceux issus des autres régions, pour la simple raison de leur appartenance à la région qui abrite l'université.

Nous pouvons également évoquer le « Mémorandum des Elites Intellectuelles de l'Est » qui réclamait aussi l'admission de la totalité des candidats originaires de cette Région au concours d'entrée dans la

\footnotetext{
${ }^{6}$ Voir à ce sujet Njawe, P.N., « Contestations », Le Messager du 15 décembre 2008, p.3 ; Belibi, J.F., « Ens de Maroua : Paul Biya accorde 100\% au Grand Nord », Mutations du 19 décembre 2008, p. 6 et Batongue, A.B., «La République des quotas : pourquoi sommes-nous Camerounais ?», Mutations du 22 décembre 2008, p. 4.
} 
toute nouvelle Ecole Normale Supérieure de Bertoua, à sa création en 2018. Il faut dire qu'avec l'avènement de cet établissement dans cette Région, les ressortissants de la Région de l'Est estiment que leur tour est arrivé, à l'instar de ce qui s'est passé à l'ENS de Maroua en 2008 avec l'admission de la totalité des «enfants du grand Nord», pour que leurs «enfants » intègrent aussi massivement cette école implantée sur leur sol. Malheureusement, cette requête qui a pourtant frappé les esprits par sa forte tonalité régionaliste, recevra une fin de non-recevoir.

Par une correspondance en date du 17 mai 2020, l'association des étudiants de l'Adamaoua dénommée "Yàwàrè bùuri sem mbe » a adressé un mémorandum au Ministre de l'Enseignement supérieur. Ce mémorandum comportant une kyrielle de griefs, visait à démontrer que les ressortissants de la Région de l'Adamaoua qui abrite l'Université de Ngaoundéré sont « lésés » aussi bien dans la répartition des postes de responsabilité que dans le recrutement des leurs en qualité d'enseignants et d'étudiants. En conséquence, les signataires de ce mémorandum demandent que les ressortissants de cette région soient davantage nommés à des postes de responsabilités dans cette université, que «leurs enfants » soient recrutés majoritairement dans les écoles de formation qui s'y trouvent et en cycle de recherche où la sélection constitue le mode de recrutement par excellence. En réaction, le Recteur de l'Université ainsi incriminée a démonté, par une suite d'illustrations à l'appui, les "contre-vérités » contenues dans les déclarations des porteurs de ce mémorandum. "En fait, le communiqué de Madame le Recteur vise à démontrer que les responsables nommés à l'Université de Ngaoundéré sont constitués en majorité des filles et fils de la Région. De même, elle a pu prouver que la plupart des étudiants dans les établissements de cette université sont originaires de la Région de l'Adamaoua, contrairement aux allégations des signataires du dernier mémorandum », indique un enseignant de l'Université de Ngaoundéré. Ces échanges épistolaires illustre l'ampleur des revendications identitaires autour de l'université au Cameroun.

Il convient de signaler que ces différents mouvements de revendication, aux apparences politiques, ont essentiellement des soubassements identitaires. Parmi les projets de leurs porteurs, se trouvent en bonne place l'insertion et l'émancipation socioprofessionnelles «des filles et fils de la région », affirme un acteur de la scène sociopolitique camerounaise. Il apparait que les memoranda constituent aussi des indicateurs du fait que les autochtones se croient souvent disposer de plus de droit que les allogènes, au regard du fait que l'université se trouve implantée sur leur "terroir». Ainsi, le témoignage de certains enseignants révèle-t-il que, avec l'ouverture de l'Université de Ngaoundéré, une certaine composante de la population autochtone de cette ville a exprimé sa crainte face au risque de contamination de «ses enfants » par la dépravation des mœurs importée par les «Gadamayo » venus du «sud ${ }^{8}$. Cette perspective illustre le raisonnement que développent encore certains parents dans les régions septentrionales du Cameroun. Selon cette conception, l'école « occidentale » apporte avec elle le modernisme et introduit des idées et des aspirations nouvelles telles que la libération et la responsabilisation des femmes. Ce qui aurait des influences négatives sur la société car, pensent les partisans de cette thèse, l'école de type classique permet l'introduction des valeurs occidentales, porteuses de conduites licencieuses et modifiant par ce fait le système traditionnel de l'éducation sexuelle.

Quant à certains enseignants allogènes en service à l'Université de Maroua, ils affirment qu'ils sont régulièrement victimes d'actes discriminatoires fondés sur la différence identitaire. Ceux-ci estiment que leurs collègues "autochtones » bénéficient de certains privilèges dus à leur appartenance à la communauté dont les terres abritent cette université. L'un d'eux indique que « la plupart des postes de responsabilité ici sont occupés par les nordistes. C'est pourquoi beaucoup d'entre-nous cherchent à rentrer au sud. La preuve, chaque année, de nombreux enseignants sudistes se font nommer dans les universités du sud ${ }^{9} \gg$, vidant ainsi l'Université de Maroua de cette catégorie de personnel. Cette situation contribue considérablement à la dégradation du climat social entre enseignants, à la

\footnotetext{
${ }^{7}$ Mot utilisé dans la langue locale pour désigner les autres comme « étrangers ».

${ }^{8}$ Terme utilisé par les populations pour désigner les populations.

${ }^{9}$ Le sud ici désigne la partie méridionale du Cameroun, à l'opposé de la partie septentrionale constituée de l'aire culturelle soudano-sahélienne.
} 
réduction du ratio enseignants/étudiants, à l'accroissement du déficit en enseignants et a pour corollaire l'affaiblissement de la capacité d'encadrement pédagogique des apprenants dans cette université. «La conséquence de cette situation est la détérioration de la qualité des enseignements à cause du brain drain que constituent les départs massifs d'enseignants compétents », poursuit-il. Ce sentiment de ségrégation socioculturelle, nourri par des suspicions et préjugés culturels, peut contribuer à mettre à mal la politique d'intégration nationale dans les universités camerounaises.

\subsubsection{Des Déclarations Comme Actions De Protestation Contre Certaines Propensions Hégémoniques}

A côté des memoranda, on peut noter des actions de protestation adressées sous forme de déclarations. Ces déclarations sonnent comme la dénonciation d'une certaine tendance hégémonique, voire expansionniste, ou comme une alerte contre les velléités de domination de certains groupes ethniques dans certaines sphères de la vie publique en général et au sein de l'université en particulier. Cette volonté de puissance d'une tribu, réelle ou supposée, a donné lieu ces dernières années à la résurgence du concept $\mathrm{d}^{\prime}$ " ethnofascisme » $[6]^{10}$. En général, la récurrente propension à ces déclarations est l'émanation de hautes autorités morales ou de membres des organisations de la société civile ou encore de groupes qui s'estiment le plus souvent lésés par les pratiques discriminatoires perpétrées au sein de l'université. Ces déclarations se font généralement par le canal des médias conventionnels et des réseaux de communication sociaux.

Parmi ces actes de protestation, nous pouvons citer l'exemple de la déclaration de l'Archevêque de Yaoundé, Grand Chancelier de l'Université Catholique d'Afrique Centrale, Monseigneur Victor Tonyé Bakot. Ce dernier indiquait en juin 2012, dans la lettre $n^{\circ}$ VTB/06/12/106/2363/enn adressée au Père Martin Brida, Doyen de la Faculté des Sciences Sociales et de Gestion, en guise de dénonciation, que les ressortissants de la Région de l'Ouest-Cameroun ${ }^{11}$ se trouvent être majoritaires, du point de vue numérique, dans cette Faculté. De même, dans cette lettre qui a défrayé la chronique, l'Archevêque métropolitain dénonce le fait que les enseignants originaires de cette même Région se trouvent également être les plus nombreux au sein de ladite Faculté. Plus qu'une indignation, cette prise de position résonne clairement comme la stigmatisation, au sein de l'université, des ressortissants de la Région indiquée. L'analyse des termes de cette correspondance donne l'impression que les enseignants de cette université se livrent plus à de manœuvres frauduleuses, qu'à la promotion des valeurs éthiques et objectives du mérite.

Suivant ce raisonnement, on peut déduire que la plus haute autorité de l'Université Catholique d'Afrique Centrale établit que si les enseignants ressortissants de la Région de l'Ouest sont les plus nombreux au sein de la Faculté des Sciences Sociales et de Gestion, et qu'il apparait que les étudiants eux aussi sont majoritairement constitués des ressortissants de la Région de l'Ouest, alors les membres de la communauté des ressortissants de cette région entretiendraient des rapports collusifs qui favoriseraient l'admission massive des leurs dans cet établissement au sein duquel l'admission se fait sur concours. En suivant ce syllogisme, l'on peut facilement parvenir à la conclusion selon laquelle ces affinités communautaires serviraient à la promotion imméritée des étudiants et des enseignants concernés au sein de cet établissement, car ces derniers bénéficieraient des faveurs de «leurs frères». Ce qui ne saurait garantir la fiabilité des enseignements, la pertinence des apprentissages et la transparence dans le système d'évaluation. En bref, ce type d'allégation jette le doute sur la qualité de la formation prodiguée dans cette institution universitaire, car elles insinuent que les conditions d'enseignement et d'apprentissage seraient viciées.

On peut également noter des dénonciations fréquentes de népotisme, de clientélisme ou de tribalisme émanant de certains groupes régionalistes qui, à l'occasion de la publication des résultats de concours

${ }^{10}$ Ce néologisme désigne, selon Mono Ndjana, la volonté de puissance d'une ethnie, ou l'expression de son désir hégémonique, qui prend soit la forme du discours théorique, soit celle d'une mêlée ouverte dans la polémique, soit celle d'une organisation systématique sous la forme du mercantilisme conquérant.

${ }^{11}$ Allusion était faite aux Bamiléké. La dénonciation sous-entendait que les membres de cette communauté ethnique bénéficiaient des faveurs ou usaient des pratiques de corruption pour s'y retrouver. 
administratifs ou des nominations de responsables dans les universités d'Etat, s'évertuent, statistiques à l'appui, à démontrer à coups de renforts médiatiques, que les ressortissants de la tribu au pouvoir dominent majoritairement la scène, en termes de représentativité. "Nous savons qu'au Cameroun, pour réussir à un concours, il faut être membre du pays organisateur ${ }^{12}$. Vous voyez qu'en général, ce sont eux qui sont en majorité admis aux concours d'entrée dans toutes les grandes écoles », s'indigne un étudiant de l'Université de Yaoundé II.

\subsection{Discussion}

\subsubsection{Des Raisons Variées Pour Justifier Les Revendications a Caractère Identitaire}

Dans l'optique de légitimer leurs actions, des raisons variées sont invoquées pour justifier les démarches entreprises par les différents mouvements de revendication identitaire.

\subsubsection{Des Raisons D'ordre Politique}

Pour justifier leur attitude revendicative, les uns ont recours à l'argument politique, voire électoraliste. «Voyez-vous, les régions septentrionales constituent à elles seules plus de $30 \%$ du vivier électoral favorable au RDPC ${ }^{13}$. C'est grâce à ces Régions que le Président Paul Biya gagne les élections depuis toujours. Ce qui fait qu'il doit nous renvoyer l'ascenseur. Nous ne pouvons pas accepter qu'on nous utilise comme du bétail électoral, bons simplement à offrir nos voix [à l'occasion des élections] », déclare un acteur sociopolitique qui s'ingénie à justifier le bien-fondé des revendications des «Elites du Grand Nord».

\subsubsection{Des raisons de défense des minorités}

Au Cameroun, certains groupes sociologiques utilisent le statut de «minorités » pour prétendre à des positions avantageuses au sein de l'université. Pour eux, la tendance hégémonique caractérisée par la domination du paysage universitaire par certaines tribus tend à réduire au silence, voire à écarter les groupes considérés comme minoritaires de l'accès à des postes de responsabilité au sein de ces institutions. Dans cette dynamique, on estime dans les arcanes de l'enseignement supérieur que les universités sont constituées à $70 \%$ par les enseignants ressortissants de la Région de l'Ouest, en l'occurrence le Bamiléké. Cette situation a amené les autorités de l'Enseignement supérieur, à l'occasion des deux premières phases de «l'opération de recrutement spécial de 2000 enseignants des universités » ordonnée par le Chef de l'Etat camerounais, à adopter une règle des quotas visant à inverser la tendance haussière des membres de cette communauté dans les sélections pour le recrutement de cette catégorie de fonctionnaires ${ }^{14}$. Cette mesure a suscité l'indignation, puis les réprobations de la part des membres de la communauté ainsi visée qui crient à l'injustice et réclament que «la priorité soit accordée au mérite, plutôt qu'aux autres considérations subjectives ou sentimentalistes », affirme un enseignant de l'Université de Dschang.

On peut aussi signaler que la levée de bouclier observée aujourd'hui à l'Université de Bamenda et à l'Université de Buea contre la «tentative de phagocytose du système anglophone par les francophones » et la « tentative de francophonisation de ces universités ${ }^{15}$ d'obédience anglosaxonne, participe non seulement de cette dynamique de dénonciation, mais également des mouvements de revendication identitaire au Cameroun. De vives protestations se sont élevées au sein et en dehors de ces universités, réclamant l'interdiction de l'admission des étudiants et enseignants francophones dans «leurs» établissements. Ce mouvement de protestation est d'ailleurs l'un des éléments déclencheurs de la crise sociopolitique qui sévit depuis 2016 dans les Régions du Nord-

\footnotetext{
12 Terme utilisé par les autres communautés du Cameroun pour désigner les membres de l'aire culturelle FangBeti, à laquelle appartient l'actuel Chef de l'Etat camerounais, exprimant l'idée que ces derniers sont les détenteurs du pouvoir politique dont ils useraient et abuseraient.

${ }^{13}$ Le RDPC est le parti au pouvoir au Cameroun.

${ }^{14}$ Cette mesure vise à établir l'équilibre entre les différentes composantes socioculturelles du Cameroun.

${ }^{15}$ Ces expressions sont devenues le crédo des mouvements de protestation et un mot d'ordre des manifestations dans ces deux Régions depuis le 21 novembre 2016, date du déclenchement de la « crise anglophone ».
} 
Ouest et du Sud-Ouest, et qui est aujourd'hui désignée par la formule «crise anglophone ». Les conditions d'enseignement/apprentissage se trouvent ainsi dégradées dans ces deux universités, car les cours sont régulièrement perturbés, le rythme académique troublé, les étudiants et les enseignants régulièrement visés par les attaques des « sécessionnistes ${ }^{16}$.

Il faut dire que ce mouvement de protestation vise essentiellement à favoriser le recrutement massif d'enseignants anglophones et l'admission de facto des étudiants de ces deux régions dans les écoles au sein desquelles l'entrée se fait sur concours. En effet, en limitant les recrutements d'enseignants et l'enrôlement des étudiants exclusivement aux candidats d'expression anglaise dans ces deux institutions universitaires, les membres de ce groupe linguistique, qui s'estiment être des minorités, espèrent voir leurs chances de recrutement s'accroître considérablement, car les francophones, qui constituent à eux seuls environ $80 \%$ de la population camerounaise, seraient d'office éliminés.

\subsubsection{Des Raisons De Protection Des Autochtones}

D'autres enfin se servent de leur qualité d'autochtones pour prétendre que « les terres de leurs parents ont servi à l'implantation de l'université », donc qu'en retour, ils doivent «bénéficier en priorité des avantages que pourrait procurer cette structure », indique un étudiant de l'Ecole Normale Supérieure de Bertoua, originaire de la Région de l'Est qui abrite ladite école. Dans ce registre, on entend régulièrement des voix s'élever, soit individuellement soit collectivement, pour réclamer des avantages inhérents au droit d'appartenance « au terroir qui abrite l'université ».

$\mathrm{Au}$ final, les raisons politiques, de minorités et d'autochtonie peuvent être considérées comme les justificatifs à cette montée de revendications identitaires qui ne manquent pas de toucher l'espace universitaire. Dans cette dynamique, la question que cette situation inspire est celle de savoir quels sont les effets d'une telle propension aux revendications sur les conditions d'enseignement et d'apprentissage au sein de ce haut lieu de production et de diffusion des savoirs.

\subsubsection{De Réelles Menaces Sur Les Conditions D'enseignement Et D’apprentissage}

Il est désormais clair que certaines attitudes et pratiques sociétales inductrices de clivages identitaires perpétrées au sein de la société, étendent leurs tentacules jusqu'au niveau de l'espace universitaire au Cameroun et ont une influence plus ou moins grandes sur les conditions d'enseignement et d'apprentissage. S'il est vrai que l'ampleur de ce phénomène n'atteint pas encore des proportions alarmantes, on peut toutefois s'inquiéter des risques qu'il fait planer sur la coexistence pacifique entre les membres de différentes communautés. Cependant, cette propension locale à l'homogénéisation culturelle constitue une véritable menace à la dynamique d'intégration sociale et académique des étudiants et des enseignants dans les universités camerounaises, car elle contribue à la soustraction du moins de façon symbolique et psychologique - d'une frange de camerounais du processus de transmission et d'acquisition des savoirs.

Ceci se manifeste par exemple à l'Université de Bamenda et à l'Université de Buea à travers la réclamation, par une frange des élites et de certains acteurs universitaires régionaux, de l'exclusion des francophones du processus d'enseignement et l'exigence de l'exclusivité de la langue anglaise comme unique langue d'enseignement. Ces attitudes, dans un pays bilingue français-anglais et dans un monde multipolaire, limitent considérablement les chances des lauréats de ces universités d'accéder aux enseignements de qualité, donc à la modernité académique, à la transversalité scientifique et à l'universalité heuristique. A ce propos, nous pensons avec Calvet [7] que : «Le plurilinguisme, par la pluralité et la diversité dont il témoigne, par la coexistence qu'il impose, par les échanges qu'il permet, peut être facteur de partage, de convivialité, d'ouverture sur les autres. " Apprends une langue, tu éviteras une guerre », dit un proverbe arabe, et ces quelques mots résonnent comme un programme ».

En outre, le phénomène de « listes », qui met en évidence les interventions des élites régionales et vise à faire inscrire des candidats «non méritants » dans des écoles dans lesquelles l'admission se fait sur

\footnotetext{
${ }^{16}$ Des enseignants et étudiants sont régulièrement kidnappés aussi bien dans l'enceinte qu'en dehors de ces deux universités et d'autres ont même été assassinés.
} 
concours, induit des biais, instaure des injustices, voire des inégalités dans le processus de recrutement. Il participe par ricochet à galvauder le processus enseignement/apprentissage à l'université dont le fondement pédagogique est pourtant la compétitivité, l'objectivité et le mérite. Ces attitudes qui limitent considérablement l'objectivité et induisent souvent des irrégularités dans le processus d'admission des étudiants et de recrutement des enseignants à l'université, compromettent les conditions de vivre-ensemble entre les citoyens d'un même pays. Par ce fait, des enseignants et des étudiants compétents et performants sont quelquefois éliminés au profit de candidats de qualité douteuse. En conséquence, ces pratiques déteignent sur l'efficacité des conditions d'enseignement et d'apprentissage : elles altèrent gravement la pertinence des enseignements, dégradent considérablement la qualité des apprentissages et affectent durement l'efficacité des produits issus de ces universités. Cet état de choses est à l'origine de la baisse du niveau des diplômés de l'enseignement supérieur si souvent décriée au Cameroun et génère souvent des tensions intercommunautaires entre acteurs du système universitaire. Les communautés qui s'estiment lésées se sentant frustrés, marginalisés. Ce qui laisse planer de réelles suspicions dans le corps de l'enseignement supérieur et des menaces sur le climat social dans les universités d'Etat.

Dans une enquête menée sur la qualité des lauréats issus de la première promotion des étudiants de l'Ecole Normale Supérieure de Maroua, Bachir Bouba [8] affirme que les compétences de ces derniers comportent des lacunes car «certains ont un background insuffisant». Les résultats de l'analyse de ce chercheur permettent d'établir une relation de cause à effet entre les interventions à caractère communautariste des élites du Grand Nord qui ont permis le recrutement massif des étudiants originaires de cette région en faisant fi des conditions d'encadrement ${ }^{17}$ des apprenants, de leurs compétences réelles (couplées à la mauvaise application du système LMD) et le déficit du processus enseignement/apprentissage dans la formation de cette cohorte d'apprenants. Ces conséquences sont à l'origine des compétences pédagogiques « moins satisfaisantes » auxquels lesdits résultats sont parvenus [8].

\section{CONCLuSion}

Cette réflexion nous a permis de voir qu'au sein d'une société multiculturelle comme le Cameroun, les clivages culturels et les replis identitaires observés font irruption dans les arcanes des universités et fragilisent les conditions d'enseignement et d'apprentissage. Lorsqu'on connait l'influence de l'éducation comme moteur de la croissance et lorsqu'on sait que la cohabitation des cultures est souvent source de conflit latent [9], voire de crises en milieu éducatif, on peut comprendre pourquoi certains groupes qui s'estiment "lésés », «marginalisés » et "frustrés » revendiquent la prise en considération de leurs spécificités culturelles dans le partage des privilèges liés à l'implantation de l'Université dans leur région d'origine.

Il convient d'indiquer que cette tendance au repli identitaire apparait comme une espèce de rempart face à l'adversité des autres communautés en quête de positionnement hégémonique. Il représente également une sorte de bouclier contre l'hostilité, voire l'agressivité traumatique du monde extérieur. La recrudescence de ce phénomène révèle la montée en puissance, au sein de différentes communautés au Cameroun, de ce que nous appelons le syndrome de l'angoisse identitaire [10]. Ce syndrome se manifestant par la peur croissante des uns et des autres de se voir aliéner et perdre leur identité, ainsi que des privilèges qui y sont rattachés. Les communautés ont tendance à se replier sur elles-mêmes afin d'exorciser leurs phobies face aux incertitudes qu'impose un monde de plus en plus complexe, concurrentiel et aux prises avec diverses formes de discrimination, d'intolérance, d'extrémisme et d'injustice.

En dépit des failles observées dans le processus d'intégration des différentes composantes sociologiques et qui entraînent l'irruption des revendications communautaristes au sein des universités camerounaises, il serait hâtif de conclure que les conditions d'enseignement et d'apprentissage se

\footnotetext{
${ }^{17}$ Les conditions d'encadrement étaient extrêmement limitées dans cette université du fait que cette institution nouvellement créée ne disposait pas encore d'infrastructures propres et adéquates pour contenir un flux aussi élevé d'étudiants.
} 
trouvent complètement dans un état de dégradation avancée. Toutefois, il convient d'y prendre garde, au regard de la situation de déliquescence dans laquelle sont plongées actuellement les Universités de Bamenda et de Buea, dont on sait que l'origine des problèmes qu'elles vivent aujourd'hui est d'ordre identitaire. C'est donc dire que les conditions d'enseignement et d'apprentissage dépendent en grande partie de la qualité de l'intégration des différentes sensibilités socioculturelles dans les universités camerounaises.

Dans l'optique de juguler les menaces qui planent sur l'intégration des différentes composantes sociologiques dans les universités camerounaises et de favoriser l'amélioration des conditions d'enseignement et d'apprentissage, nous proposons le développement d'un mécanisme de médiation interculturelle, en même temps que l'effort de construction d'une conscience nationale et d'un sentiment d'appartenance à une « communauté universitaire » qui se veut universelle. Cela conduirait à une autorégulation des comportements, résultat d'un changement des schèmes mentaux, et aboutirait à dissocier les discours intégrationnistes, tenus par les hommes politiques, des pratiques ségrégationnistes observées actuellement dans plusieurs sphères de la société camerounaise, $\mathrm{y}$ compris au sein des universités. La fonction principale de cette dynamique faîtière serait de promouvoir l'ouverture et le dialogue interculturels au sein des communautés, tout en protégeant les valeurs patrimoniales propres à chaque groupe. Cette approche permettrait d'engager acteurs sociaux et responsables universitaires à assurer et à assumer l'inclusion, l'émancipation et la pleine participation de toutes les entités sociologiques au processus d'édification d'une université dénuée de toute tendance ségrégationniste fondée sur l'origine culturelle des différents acteurs. Ainsi, elle contribuerait significativement à œuvrer à la nécessaire co-construction d'une citoyenneté inclusive et d'une identité partagée à travers la prise en compte des spécificités culturelles de chaque groupe, ce qui favoriserait en conséquence l'amélioration des conditions d'enseignement et d'apprentissage. Cette entreprise doit essentiellement reposer sur la prise de conscience que : «Les intégrismes en tant que phénomènes culturels naissent du réductionnisme qui fait d'une culture la culture; ils excluent donc de la société les autres cultures. Le dogmatisme et l'autoritarisme monoculturel sont intolérants. Les conséquences de cette méconnaissance du multiculturalisme sont la discrimination et la violence » [11].

\section{REFERENCES}

[1] VAN DER MAREN, J-M., Méthodes de recherche pour l'éducation. $2^{\mathrm{e}}$ édition, Montréal : Les Presses de l'Université de Montréal, 1996.

[2] BARDIN L., L'analyse de contenu, Paris : PUF, 2007.

[3] L-C. BOYOMO ASSALA, «Nation, Etat : discours intégrationnistes et pratiques ségrégationnistes, In SIMO, D. (ed.), Constructions identitaires en Afrique : enjeux, stratégies et conséquences, pp. 134-173, Yaoundé : Editions Clé, 2006.

[4] YODOU SIBEUDEU, A.P., Justice politique et prévention des conflits dans les sociétés pluriethniques : cas de la politique de l'équilibre régional au Cameroun, Mémoire de Master II en Sciences Sociales. Inédit, Université Catholique d'Afrique Centrale, 2011.

[5] H.-de-P. POKAM, «La participation des universitaires au processus de construction/reconstruction de l'espace public au Cameroun », L'Anthropologue africain, Vol. 17, $\mathrm{n}^{\circ} 1$ \& 2, pp. 81-116, 2010.

[6] H. MONO NDJANA, «De l'ethnofascisme dans la littérature politique camerounaise », Peuples noirs, peuples africains, [Revue bimestrielle], $10^{\mathrm{e}}$ année, $\mathrm{n}^{\circ} 55-58$, pp. 112-130, 1987.

[7] L-J. CALVET, "Identités et plurilinguisme", Trois espaces linguistiques face aux défis de la mondialisation, Actes du colloque international organisé par l'AUF, 20-21 mars 2001, Paris : AUF, pp. 147-160, 2001.

[8] BACHIR BOUBA, «De la théorie à la pratique : l'évaluation de la mise en œuvre du système LMD à l'Université de Maroua et la qualité de la formation », La Recherche en Education, n ${ }^{\circ} 11$, pp. 32-47, 2014.

[9] B. GARNIER, «La gestion des conflits interpersonnels en milieu universitaire ». Relation industrielle. 38(2), pp. 277-296, $1983 \mathrm{https}: / /$ doi/10.7202/029353ar.

[10] MAMA CHANDINI, «Multiculturalisme, intégration socioculturelle des étudiants et enseignement/apprentissage en milieu universitaire au Cameroun », In Bachir Bouba. (Dir.), Education, formation et apprentissage en contexte multiculturel camerounais, Douala : Editions Cheikh Anta Diop, pp. 57-93, 2020. 
L'université Camerounaise Face Aux Mouvements De Revendications Identitaires [Cameroon's universities and identity-based movements]

[11] G.H. VASQUEZ, «Communication interculturelle pour "démocratiser la Démocratie" », Trois espaces linguistiques face aux défis de la mondialisation, Actes du colloque international organisé par l'AUF, 2021 mars 2001, Paris : AUF, pp. 129-146, 2001.

\section{AUTHOR'S BIOGRAPHY}

Mama Chandini, est titulaire d'un Doctorat/Ph.D en Sciences de l'Education et enseignant-chercheur au Département des Sciences de l'Education de l'Ecole Normale Supérieure de Bertoua, Université de Ngaoundéré au Cameroun, où il est Coordonnateur de la section Conseillers d'Orientation. Parallèlement, il occupe actuellement la fonction de Chef de Division des Affaires Administratives et Financières à l'Université de Dschang. Ses centres d'intérêt portent sur les questions d'orientationconseil, de professionnalisation de l'enseignement, de développement des compétences et d'innovation en formation.

Citation: Mama Chandini. "L'université Camerounaise Face Aux Mouvements De Revendications Identitaires [Cameroon's universities and identity-based movements]" International Journal of Humanities Social Sciences and Education (IJHSSE), vol 8, no. 5, 2021, pp. 64-75. doi: https://doi.org/10.20431/23490381.0805008.

Copyright: () 2021 Authors. This is an open-access article distributed under the terms of the Creative Commons Attribution License, which permits unrestricted use, distribution, and reproduction in any medium, provided the original author and source are credited. 